\title{
Prognostication in the era of a new stressor for myocardial perfusion imaging
}

\author{
Wanda Acampa, MD, PhD, ${ }^{a}$ Marco Salvatore, $M D,{ }^{b}$ and Alberto Cuocolo, $\mathrm{MD}^{\mathrm{c}}$ \\ a Institute of Biostructures and Bioimaging, National Council of Research, Naples, Italy \\ ${ }^{\mathrm{b}}$ IRCCS SDN, Naples, Italy \\ c Department of Advanced Biomedical Sciences, University Federico II, Naples, Italy
}

Received Jan 13, 2015; accepted Jan 13, 2015

doi: $10.1007 / \mathrm{s} 12350-015-0075-x$

\section{See related article, pp. 1214-1221}

Myocardial perfusion imaging (MPI) both with exercise and vasodilator stress has been widely used for diagnostic and prognostic evaluation of patients with suspected or known coronary artery disease (CAD). Despite diagnosis remains a primary indication for CAD testing, risk stratification has become important in patients management. In fact, the established concept of guided care in the present era is linked to the concept of risk stratification using different imaging modalities. Accordingly, the optimal improvement in outcome may be achieved by linking high-risk imaging measures to risk-reducing therapies. ${ }^{1}$ It has been demonstrated that among the different noninvasive imaging modalities available in the setting of CAD, MPI with single-photon emission computed tomography (SPECT) provides information about physiologic significance of flow-limiting disease leading to a global estimation of risk for major adverse cardiac events. ${ }^{2}$ Different semi-quantitative or quantitative measures of myocardial perfusion abnormalities on stress-rest MPI are related to the risk of cardiac event over time. A wealth of clinical research has shown that the extent and severity of stress-induced perfusion defects are the powerful and predominant predictors of most post-MPI outcomes. ${ }^{3-5}$ As risk of adverse events increases with the extent and severity of MPI abnormalities, patients in high-risk scan categories

Reprint requests: Wanda Acampa, $\mathrm{MD}, \mathrm{PhD}$, Institute of Biostructures and Bioimaging, National Council of Research, Naples, Italy; acampa@unina.it

J Nucl Cardiol 2015;22:1222-4.

$1071-3581 / \$ 34.00$

Copyright (c) 2015 American Society of Nuclear Cardiology. result at greatest risk for future cardiac events. It has also been demonstrated that post-stress left ventricular ejection fraction (LVEF) provides incremental information over extent and severity of myocardial perfusion defects for the prediction of cardiac death. ${ }^{6}$ However, while LVEF predicts the risk of cardiac death, only quantitative measures of ischemia at MPI can identify the patients that will accrue a survival benefit from revascularization compared to medical therapy. ${ }^{7,8}$ Despite this certainty reached, relatively recent literature aimed to improve the knowledge on the relationship between MPI measures and prognosis, covering all the different aspects of myocardial perfusion and function quantification. Shaw et $\mathrm{al}^{9} \mathrm{dem}-$ onstrated that CAD risk may be optimally estimated by use of a combination of resting SPECT myocardial perfusion, reflecting a patient's burden of disease, and SPECT with provocative ischemia.

\section{CLINICAL USE OR REGADENOSON MPI}

Regadenoson is at present the only selective adenosine $\mathrm{A}_{2 \mathrm{~A}}$ receptor agonist commercially available for stress MPI, and its development has contributed significantly to understanding the adenosine action mechanisms and its vasodilator effect on the coronary circulation. Regadenoson low affinity for the $A_{2 A}$ receptor explains its short duration of action $(\leq 5 \mathrm{~min}$ utes), an advantage as it avoids unnecessarily prolonged vasodilation. The duration of action of regadenoson is adequate to allow for adequate extraction of the tracer by the myocardium. ${ }^{10}$ In experimental data, peak increase in coronary blood flow by regadenoson seems to be similar to that induced by adenosine, but lower concentrations of regadenoson are needed for any level of blood flow increase. ${ }^{11}$ Other studies demonstrated that regadenoson leads to significantly greater coronary arteriolar vasodilation and consequently higher myocardial blood flow than adenosine causing vasodilation, ${ }^{12}$ making regadenoson a potentially more effective 
vasodilator agent with more sustained hyperemia compared to adenosine. ${ }^{13}$ The major features of regadenoson as pharmacological stress agent are that dose calculation is not required, no continuous administration requested obviating the need for an infusion pump, and a higher patient's side-effect profile tolerability. Improved tolerability to stress and simplification of stress-testing protocol are the major advantages associated with the clinical use of regadenoson. Accordingly, the demonstrated safety associated to its use in patients with chronic pulmonary disease as well as in patients with renal and liver disease affords greater availability of stress MPI to virtually all patients with suspected or known CAD. ${ }^{14}$ Multicenter randomized trials have demonstrated that regadenoson is non-inferior to adenosine for the detection of reversible perfusion defects. ${ }^{15,16}$ In particular, applying a quantitative analysis of SPECT MPI data, regadenoson provided similar results as adenosine regarding the total LV perfusion defect size (PDS). Importantly, it has been demonstrated that regadenoson induces perfusion abnormalities similar to those observed with adenosine irrespective of patient clinical characteristics or cardiac history. ${ }^{17}$ Thus, it has been suggested that regadenoson provides comparable diagnostic and prognostic SPECT information to that obtained with adenosine across heterogeneous patient populations. ${ }^{15}$ More recent data reported that patients with normal regadenoson MPI (normal perfusion and LV function) have a low incidence of major adverse cardiovascular events during a 2-year follow-up, similar to those studied with adenosine in a cohort of propensity-matched patients. ${ }^{18}$ Low risk associated with normal regadenoson SPECT MPI provided important knowledge regarding patient management after testing, in respect of one of the principles of risk stratification that is to defining clearly patient cohort with improved outcome. Moreover, patients with a normal heart rate response had a relatively low-annualized total mortality and heart rate response determined a net reclassification improvement when compared to traditional MPI findings. ${ }^{17,19}$ The use of the change in heart rate in response to regadenoson provides the opportunity to better stratify patients in the setting of both normal and abnormal MPI findings.

In this issue of the journal, Hage et $\mathrm{al}^{20}$ evaluated the prognostic value of automated quantification of perfusion abnormalities by regadenoson SPECT MPI. The authors studied 1,400 patients referred to MPI with regadenoson as stress test. Patients with abnormal perfusion imaging at quantitative analysis (\% abnormal myocardium) were followed within 2 years, for the occurrence of outcome events (primary outcome) including cardiac death, myocardial infarction, and coronary revascularization procedures. The results showed that cardiovascular risk increases according to the extent and severity of myocardial perfusion defect. In particular, it has been observed a graded relationship such that as PDS worsened event rate significantly increased, both considering primary outcome (composite endpoints) as its individual components. The same relationship has been observed between $\%$ ischemic myocardium and the outcome endpoints. More important, both PDS and \% ischemic myocardium demonstrated an independent association with the primary outcome. In the study of Hage et al, ${ }^{20}$ multivariate analysis helped to define the independent contribution of any set of variables in estimating adverse cardiac events providing a powerful contribution to define the prognostic value of regadenoson SPECT MPI in a non-selected patient population. To completion of MPI results evaluation, abnormal LV function was independently associated with an increased risk of cardiac death. Hence, the study of Hage et $\mathrm{al}^{20}$ is important to integrate and delineate the clinical use of new pharmacological stress agents also for prognostication of patients with suspected or known CAD. The powerful of these data might have important clinical implications considering that each of the prognostic variables obtained by a specific imaging modality should confer a determinant contribution in guiding patient management.

Regadenoson has beginning to be used also for positron emission tomography (PET) MPI. Published studies validated the use of regadenoson for quantitative absolute quantification of myocardial blood flow and coronary flow reserve by ${ }^{82} \mathrm{Rb}$ PET and demonstrated that regadenoson is a suitable alternate to dipyridamole or adenosine stress testing in clinical practice. ${ }^{21-23}$ In particular, regadenoson-induced stress myocardial blood flow and myocardial blood flow reserve at ${ }^{82} \mathrm{Rb}$ PET MPI resulted quantitatively equivalent to dipyridamole in patients with normal PET images. ${ }^{21}$ Including also patients with abnormal findings it has been observed an overall high degree of correlation between the paired dipyridamole and regadenoson stress perfusion, changes in stress and rest perfusion, LVEF, LV volumes, and change between stress and rest function. ${ }^{22}$ Accordingly, regadenoson stress PET MPI showed a good diagnostic accuracy in the detection of obstructive CAD. ${ }^{23}$ Recently, it has been demonstrated the evidence for the use of regadenoson as the primary agent for fractional flow reserve measurement ${ }^{24,25}$ also in consideration of its effective hyperemia. However, despite the higher myocardial blood flow induced by regadenoson compared to adenosine, ${ }^{7}$ these two stressors produce similar pressure-derived fractional flow reserve. ${ }^{26}$ Probably, the next step should be to verify the prognostic power of regadenoson PET MPI also in comparison to adenosine to better elucidate the capabilities of this new 
tracer in evaluating the absolute quantification of myocardial blood flow and coronary flow reserve. The acquired evidence will help us to better address to nuclear-guided patient management strategies.

\section{Disclosures}

The authors have indicated that they have no financial conflict of interest.

\section{References}

1. Shaw LJ, Iskandrian AE. Prognostic value of gated myocardial perfusion SPECT. J Nucl Cardiol 2004;2:171-85.

2. Nappi C, Acampa W, Pellegrino T, Petretta M, Cuocolo A. Beyond ultrasound: Advances in multimodality cardiac imaging. Intern Emerg Med 2014 [Epub ahead of print].

3. Acampa W, Petretta MP, Daniele S, Perrone-Filardi P, Petretta M, Cuocolo A. Myocardial perfusion imaging after coronary revascularization: A clinical appraisal. Eur J Nucl Med Mol Imaging 2013;40:1275-82.

4. Acampa W, Petretta M, Daniele S, Del Prete G, Assante R, Zampella $\mathrm{E}$, et al. Incremental prognostic value of stress myocardial perfusion imaging in asymptomatic diabetic patients. Atherosclerosis 2013;227:307-12.

5. Acampa W, Petretta M, Evangelista L, Daniele S, Xhoxhi E, De Rimini ML, et al. Myocardial perfusion imaging and risk classification for coronary heart disease in diabetic patients. The IDIS study: A prospective, multicentre trial. Eur J Nucl Med Mol Imaging 2012;39:387-95.

6. Sharir T, Germano G, Kang X, Lewin HC, Miranda R, Cohen I, et al. Prediction of myocardial infarction versus cardiac death by gated myocardial perfusion SPECT: Risk stratification by the amount of stress-induced ischemia and the poststress ejection fraction. J Nucl Med 2001;42:831-7.

7. Hachamovitch R, Hayes SW, Friedman JD, Cohen I, Berman DS. Comparison of the short-term survival benefit associated with revascularization compared with medical therapy in patients with no prior coronary artery disease undergoing stress myocardial perfusion single photon emission computed tomography. Circulation 2003;107:2900-7.

8. Shaw LJ, Berman DS, Maron DJ, Mancini GB, Hayes SW, Hartigan PM, et al. Optimal medical therapy with or without percutaneous coronary intervention to reduce ischemic burden: Results from the Clinical Outcomes Utilizing Revascularization and Aggressive Drug Evaluation (COURAGE) trial nuclear substudy. Circulation 2008;117:1283-91.

9. Shaw LJ, Hendel RC, Heller GV, Borges-Neto S, Cerqueira M, Berman DS. Prognostic estimation of coronary artery disease risk with resting perfusion abnormalities and stress ischemia on myocardial perfusion SPECT. J Nucl Cardiol 2008;15:762-73.

10. Glover DK, Okada RD. Myocardial kinetics of Tc-MIBI in canine myocardium after dipyridamole. Circulation 1990;81:628-37.

11. Trochu JN, Zhao G, Post H, Xu X, Belardinelli L, Belloni FL, et al. Selective A2A adenosine receptor agonist as a coronary vasodilator in conscious dogs: Potential for use in myocardial perfusion imaging. J Cardiovasc Pharmacol 2003;41:132-9.

12. Gao Z, Li Z, Baker SP, Lasley RD, Meyer S, Elzein E, et al. Novel short-acting A2A adenosine receptor agonists for coronary vasodilation: Inverse relationship between affinity and duration of action of A2A agonists. J Pharmacol Exp Ther 2001;298:209-18.
13. Al Jaroudi W, Iskandrian AE. Regadenoson: A new myocardial stress agent. J Am Coll Cardiol 2009;54:1123-30.

14. Thomas GS, Tammelin BR, Schiffman GL, Marquez R, Rice DL, Milikien D, et al. Safety of regadenoson, a selective adenosine A2A agonist, in patients with chronic obstructive pulmonary disease: A randomized, double-blind, placebo-controlled trial (RegCOPD trial). J Nucl Cardiol 2008;15:319-28.

15. Iskandrian AE, Bateman TM, Belardinelli L, Blackburn B, Cerqueira MD, Hendel RC, et al. Adenosine versus regadenoson comparative evaluation in myocardial perfusion imaging: Results of the ADVANCE phase 3 multicenter international trial. J Nucl Cardiol 2007;14:645-58.

16. Cerqueira MD, Nguyen P, Staehr P, Underwood SR, Iskandrian AE. Effects of age, gender, obesity, and diabetes on the efficacy and safety of the selective A2A agonist regadenoson versus adenosine in myocardial perfusion imaging: Integrated ADVANCE-MPI trial results. J Am Coll Cardiol Cardiol Imaging 2008;1:307-16.

17. Mahmarian JJ, Peterson LE, Xu J, Cerqueira MD, Iskandrian AE, Bateman TM, et al. Regadenoson provides perfusion results comparable to adenosine in heterogeneous patient populations: A quantitative analysis from the ADVANCE MPI trials. J Nucl Cardiol 2014 [Epub ahead of print].

18. Iqbal FM, Hage FG, Ahmed A, Dean PJ, Raslan S, Heo J, et al, Comparison of the prognostic value of normal regadenoson with normal adenosine myocardial perfusion imaging with propensity score matching. JACC Cardiovasc Imaging 2012;5:1014-21.

19. Hage FG, Dean P, Iqbal F, Heo J, Iskandrian AE. A blunted heart rate response to regadenoson is an independent prognostic indicator in patients undergoing myocardial perfusion imaging. J Nucl Cardiol 2011;18:1086-94.

20. Hage FG, Ghimire G, Lester D, Mckay J, Bleich S, El-Hajj S, et al. The prognostic value of regadenoson myocardial perfusion imaging. J Nucl Cardiol (in press).

21. Goudarzi B, Fukushima K, Bravo P, Merrill J, Bengel FM. Comparison of the myocardial blood flow response to regadenoson and dipyridamole: A quantitative analysis in patients referred for clinical 82Rb myocardial perfusion PET. Eur J Nucl Med Mol Imaging 2011;38:1908-16.

22. Cullom SJ, Case JA, Courter SA, McGhie AI, Bateman TM. Regadenoson pharmacologic rubidium-82 PET: A comparison of quantitative perfusion and function to dipyridamole. J Nucl Cardiol 2013;20:76-83.

23. Hsiao E, Ali B, Blankstein R, Skali H, Ali T, Bruyere J Jr, et al. Detection of obstructive coronary artery disease using regadenoson stress and $82 \mathrm{Rb}$ PET/CT myocardial perfusion imaging. J Nucl Med 2013;54:1748-54.

24. Nair PK, Marroquin OC, Mulukutla SR, Khandhar S, Gulati V, Schindler JT, Lee JS. Clinical utility of regadenoson for assessing fractional flow reserve. JACC Cardiovasc Interv 2011;4:1085-92.

25. Prasad A, Zareh M, Doherty R, Gopal A, Vora H, Somma K, et al. Use of regadenoson for measurement of fractional flow reserve. Catheter Cardiovasc Interv 2014;83:369-74.

26. Arumugham P, Figueredo VM, Patel PB, Morris DL. Comparison of intravenous adenosine and intravenous regadenoson for the measurement of pressure-derived coronary fractional flow reserve. EuroIntervention 2013;8:1166-71. 